SHORT REPORT

\title{
The significance of elevated CSF lactate
}

\section{S L Chow, Z J Rooney, M A Cleary, P T Clayton, J V Leonard}

The final diagnosis of 158 patients who had a cerebrospinal fluid (CSF) lactate concentration greater than $2 \mathrm{mmol} / \mathrm{l}$ was ascertained. The conditions included seizures, inflammatory changes, and proven metabolic disorders. For the diagnosis of congenital lactic acidoses, CSF lactate should ideally be measured in a seizure free patient after any acute illness.

A s blood lactate concentrations are influenced by several factors, cerebrospinal fluid (CSF) lactate concentrations are widely used in the diagnosis of congenital lactic acidoses. ${ }^{12}$ However, CSF lactate may also be raised in association with many other illnesses including seizures, ${ }^{3}$ meningitis, ${ }^{4}$ encephalitis, and cerebral ischaemia. In order to determine the value of measuring CSF lactate, we have reviewed the records of 158 children who attended a tertiary referral hospital with concentrations greater than or equal to $2 \mathrm{mmol} / \mathrm{l}$.

\section{METHODS}

In this study, CSF lactate measurements were reviewed for the 10 year period of 1992-2002. The medical records of those patients with values equal to or greater than $2 \mathrm{mmol} / \mathrm{l}$ were retrieved. For patients in whom more than one CSF lactate measurement was taken, the highest value was used. Additional details were also recorded, including the patient's condition at the time of the lumbar puncture, the presence of seizures, haemodynamic status, other metabolic investigations, and the final diagnosis. The project was registered with the Research and Development office and the patient details anonymised.

\section{RESULTS}

During the study period, CSF lactate was measured on 2268 occasions. Of these measurements, 414 (18\%) values from 267 patients were greater than or equal to $2 \mathrm{mmol} / \mathrm{l}$. We were able to review the records of $158(59 \%)$ patients. CSF lactate concentrations for patients grouped according to diagnosis are shown in fig 1 .

\section{Metabolic disorders}

Forty five patients (28\%) had a metabolic disorder (37 respiratory chain disorder, five pyruvate dehydrogenase deficiency, two Krabbe's leukodystrophy, and one biotinidase deficiency).

\section{Seizures}

In 24 patients $(15 \%)$ the raised CSF lactate value was attributed solely to seizure activity occurring within 3 days of the lumbar puncture.

\section{Intra-cranial inflammation}

Twenty nine patients (18\%) had encephalitis, meningitis, or cerebral inflammation. Nine $(6 \%)$ had cerebral ischaemia.

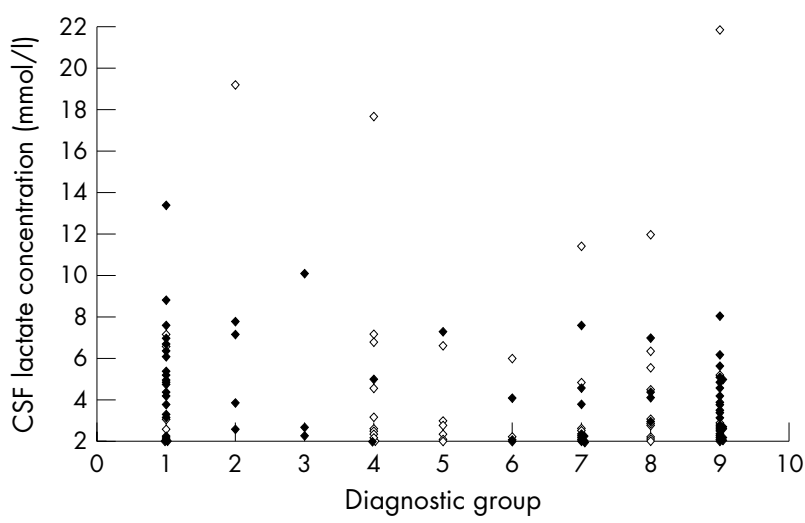

Figure 1 CSF lactate concentrations in different diagnostic groups. Open symbols: sample in acute illness. Closed symbols: sample in chronic illness. 1: Respiratory chain disorders; 2: pyruvate dehydrogenase deficiency; 3: other metabolic disorders (see text); 4: infection/inflammation; 5: ischaemia; 6: malignancy; 7: seizures; 8: multiple aetiology; 9: unknown.

\section{Malignancy}

Six $(4 \%)$ of the patients had malignancy. In five patients the malignancy was cerebral or meningeal in origin. The other patient had an adrenal neuroblastoma with cerebral metastases.

\section{Other causes}

Several patients had more than one cause for raised CSF lactate concentrations. Of the 36 patients with high CSF lactate values within 3 days of active seizures, 12 patients had other diagnoses which are likely to have contributed to the result as follows: three had a respiratory chain defect, six an inflammatory process (two meningitis, two encephalitis, one SLE, and one post-streptococcal acute disseminated encephalomyelitis), one cerebral ischaemia, and two other metabolic disorders (one Gaucher's disease and one Menke's syndrome). One patient with a posterior fossa medulloblastoma developed postoperative meningitis and another with tuberculous meningitis developed a middle cerebral artery infarct with both of these factors contributing to the raised CSF lactate values of 5.6 and $6.4 \mathrm{mmol} / \mathrm{l}$, respectively.

In the remaining $45(28 \%)$ patients, no obvious cause was found for the increased CSF lactate measurement. One 19 day old patient was acutely unwell with multi-organ failure; an infection screen, urine organic acids, plasma amino acids, muscle histology, and respiratory chain enzymes failed to identify the cause of a raised CSF lactate value of $21.9 \mathrm{mmol} / \mathrm{l}$. Twenty nine of the 45 patients had normal metabolic investigations including muscle histology and respiratory chain enzyme assay. Eight of these had an illness consistent with congenital lactic acidosis and, although the investigations failed to confirm this, a metabolic disorder was

Abbreviation: CSF, cerebrospinal fluid 
still likely. Sixteen patients had metabolic investigations that did not include a muscle biopsy. In three of these patients, definitive diagnoses were made of illnesses not known to cause raised CSF lactate values (vanishing white matter disease, juvenile idiopathic arthritis with papillitis, and hereditary sensory motor neuropathy type VI). However, the values in these patients were only marginally raised (22.07).

\section{DISCUSSION}

Great Ormond Street Hospital is a tertiary referral children's hospital and the specialist nature of the hospital will have influenced the diagnoses and frequency of raised CSF lactate values. Furthermore, only $60 \%$ of the notes were reviewed. Nevertheless, the results show that in the patients investigated a CSF lactate value greater than $2 \mathrm{mmol} / \mathrm{l}$ is not uncommon as $18 \%$ of all values were above this concentration.

Our study confirms that CSF lactate concentrations can be raised in association with seizures, meningitis or encephalitis, cerebral ischaemia, malignancy, and other metabolic disorders and is not specific for congenital lactic acidoses.

Of patients who were acutely unwell at the time of the lumbar puncture, only $9 \%$ were found to have congenital lactic acidosis, compared to $34 \%$ of patients who were in the chronic phase of their illness. CSF lactate concentration is less specific for congenital lactic acidoses in patients who are acutely unwell.

Measurement of blood lactate is often used as a first line assessment of those with presumed congenital lactic acidosis. However, it is recognised that blood lactate levels are notoriously variable, being affected by many factors including muscle movement. Furthermore, the CSF lactate level may be increased when the plasma or blood lactate concentration is normal in children with electron transport chain defects. ${ }^{12}$ If CSF lactate measurements are to be used to identify patients requiring further investigation for congenital lactic acidoses, the test should ideally be performed in a seizure free patient during the chronic phase of the illness, but even so it should be remembered that it may be normal in those with a proven mitochondrial disorder. $^{5}$

\section{Authors' affiliations}

S L Chow, Z J Rooney, M A Cleary, P T Clayton, J V Leonard, Metabolic Unit, Great Ormond Street Hospital for Children, London, UK

P T Clayton, J V Leonard, Biochemistry, Endocrinology and Metabolism Unit, Institute of Child Health, University College London, London, UK

Competing interests: none declared

Correspondence to: Professor James Leonard, 40A Bagley Wood Road, Kennington, Oxford, OX15LY, UK; i.leonard@ich.ucl.ac.uk

Accepted 21 July 2005

\section{REFERENCES}

1 Hutchesson A, Preece MA, Gray G, et al. Measurement of lactate in cerebrospinal fluid in investigation of metabolic disease. Clin Chem 1997;43(1):158-61

2 Stacpoole PW, Bunch ST, Neiberger RE, et al. The importance of cerebrospinal fluid lactate in the evaluation of congenital lactic acidosis. J Pediatr 1999;134(1):99-102.

3 Calabrese VP, Gruemer HD, James K, et al. Cerebrospinal fluid lactate levels and prognosis in status epilepticus. Epilepsia 1991;32(6):816-21.

4 Eross J, Silink M, Dorman D. Cerebrospinal fluid lactic acidosis in bacterial meningitis. Arch Dis Child 1981;56(9):692-8

5 Smeitink JAM. Mitichondrial disorders: clinical presentation and diagnostic dilemmas. J Inherit Metab Dis 2003;26:199-207.

\section{IMAGES IN PAEDIATRICS}

\section{Muscle fibre type grouping in high resolution ultrasound}

A 5 year old boy with motor retardation presented with proximal weakness maximal in the lower limbs and generalised muscular atrophy. The deep tendon reflexes were absent. Tremor or fasciculations were not seen. The muscles of the boy were examined by a high resolution ultrasound system and real time compound imaging. The deep muscles consisted of atrophic fibres (bright areas in fig 1) with groups of hypertrophic fibres (black areas in fig 2). The analysis of the survival motor neurone (SMN) 1 gene resulted in a homozygous deletion of exon 7 and 8 confirming the diagnosis of spinal muscular atrophy.

Muscle fibres of the denervated motor unit atrophy. Reinnervation by collateral sprouting results in grouping of hypertrophic fibres with the same histochemical type, commonly called "type grouping"

Using this new ultrasound technique, ultrasound morphological

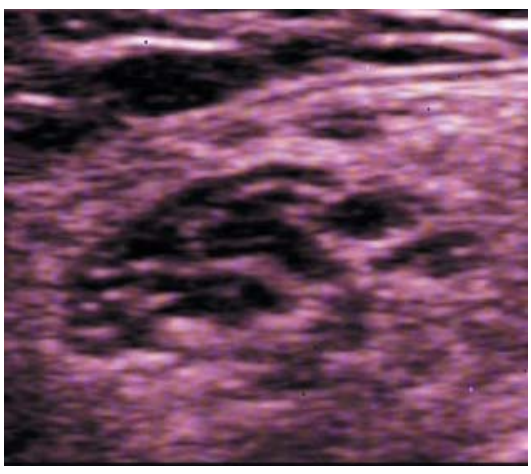

Figure 1 Cross ultrasound section of $\mathrm{m}$. vastus intermedius showing the groups of hypertrophic among the atrophic muscle fibres.

findings can be compared to histological findings.

A van Baalen, U Stephani Universitätsklinikum Schleswig-Holstein, Kiel, Germany
Figure 2 Schematic drawing of fig 1 with black areas showing the groups of hypertrophic muscle fibres.

Correspondence to: $\operatorname{Dr}$ A van Baalen, Department of Neuropediatrics, Universitätsklinikum Schleswig-Holstein, Schwanenweg 20, Kiel 24105, Germany; van.baalen@pedneuro.uni-kiel.de Competing interests: none declared 